ISSN 0103-5150

Fisioter. Mov., Curitiba, v. 26, n. 1, p. 13-23, jan./mar. 2013

Licenciado sob uma Licença Creative Commons

\title{
Facilidades e habilidades do fisioterapeuta na procura, interpretação e aplicação do conhecimento científico na prática clínica: um estudo piloto
}

\author{
Easiness and skillfulness of physical therapists \\ in searching, interpreting and applying scientific \\ knowledge in clinical practice: a pilot study
}

\author{
Priscyla Silva Queiroz ${ }^{[a]}$, Marcio José dos Santos ${ }^{[\mathrm{b}]}$ \\ [a] Acadêmica do curso de Graduação em Fisioterapia da Universidade do Estado de Santa Catarina (Udesc), Florianópolis, \\ SC - Brasil, e-mail: queiroz.pri@hotmail.com \\ [b] Ph.D. em Ciências da Reabilitação pela University of Kansas Medical Center (KUMC), professor do Departamento de \\ Fisioterapia e do Programa de Pós-Graduação do Centro de Ciências da Saúde e do Esporte (Cefid) da Universidade do \\ Estado de Santa Catarina (Udesc), Florianópolis, SC - Brasil, e-mail: marcio.santos@udesc.br
}

\section{Resumo}

Introdução: A procura e o interesse em aplicar conhecimento científico à prática fisioterapêutica tem crescido nos últimos anos. Na busca por integrar as experiências individuais vivenciadas na prática com a melhor evidência clínica disponível a partir de pesquisas sistemáticas, surge a Prática Baseada em Evidência (PBE), movimento científico que vem ganhando força na área da saúde. Entretanto, muito pouco se sabe se o fisioterapeuta clínico conhece e utiliza a PBE em seus atendimentos. Objetivo: Averiguar se os profissionais fisioterapeutas têm facilidade e habilidade em buscar literatura científica e se eles se baseiam na PBE durante sua prática clínica. Materiais e métodos: Foi realizado um estudo descritivo, com abordagem quantitativa, do qual participaram 67 profissionais fisioterapeutas atuantes na região da Grande Florianópolis. Todos responderam a um questionário online adaptado a partir do Evidence-Based Practice (EBP) Questionnaire. Resultados: A amostra consistiu em fisioterapeutas jovens, em uma faixa de idade entre 20 e 29 anos, havendo forte predominância do sexo feminino. Destes, $73,1 \%$ concluiu a graduação há menos de cinco anos e 71,6\% não possui especialidade reconhecida pelo Crefito. Os fisioterapeutas afirmaram ter uma atitude positiva sobre PBE e 
estavam interessados em aprender ou aprimorar as competências necessárias para implementar PBE. Eles notaram que precisavam aumentar o uso da evidência científica em suas práticas diárias. Conclusão: A falta de tempo foi a barreira mais importante para o uso da PBE, seguida da falta de generalização dos achados da literatura científica para sua população de pacientes e falta de fontes de informação.

Palavras-chave: Fisioterapia. Perfil profissional. Prática baseada em evidência.

\section{Abstract}

Introduction: The interest in applying scientific knowledge to physical therapy practice has grown in recent years. It has been emerged in health care the Evidence Based Practice (EBP), which is a scientific movement that looks for ways to integrate individual experiences of the physical therapy practice with the best available clinical evidence from systematic research. However, little is known about whether physical therapists (PTs) know and apply the EBP in their clinical practice. Objective: Therefore, this study aims to verify whether physical therapists are able and skillful on searching scientific literature and if they base their clinical practices on scientific literature. Materials and methods: A descriptive study, with quantitative approach was performed. Sixty-seven PTs, who work in the Greater Florianópolis district, responded a modified online questionnaire from the Evidence-Based Practice (EBP) Questionnaire. Results: The population of the interviewed PTs was mostly young, females with age range between 20 and 29 years old. Possibly because their age, 73.1\% of respondents completed their PT degree within the last five years and $71.6 \%$ have no post-graduation courses recognized by the Crefito. The PTs alleged to have a positive attitude about EBP and were interested in learning or improving their skills required to implement EBP. They agreed with the need to increase the use of scientific evidence in their daily practice. Conclusion: The lack of time was the most important barrier to the use of EBP, which was followed by the difficult in generalizing the findings of the scientific literature for patient populations, and deficient sources of information.

Keywords: Physical therapy. Professional profile. Evidence-based practice.

\section{Introdução}

Desde o seu reconhecimento como profissional legal, pela Lei n. 938 de 13 de outubro de 1969 (DOU n. 197.14.10.1969) (1), o fisioterapeuta tem buscado se integrar às equipes de saúde, mostrando seu serviço de assistência, ações de prevenção, tratamento e reabilitação, assim como de promoção em saúde. Essa assistência fisioterapêutica vem tornando-se cada vez mais complexa e variada, acrescida de novas técnicas que necessitam de fundamentação científica norteadora da prática clínica a fim de aprimorar a formação dos profissionais e consolidar a profissão.

Nos últimos anos, a procura e o interesse em aplicar conhecimento científico à prática fisioterapêutica tem crescido. É notável o crescimento dos cursos de pós-graduação em Fisioterapia e do número de doutores. Houve um crescimento significativo de pesquisadores doutores com graduação em Fisioterapia, saltando de um acumulado de 57 pesquisadores em 1998 para 573 em 2008, o que gerou um aumento considerável nas produções científicas (2).
0 aumento significativo nas produções científicas na área de fisioterapia e o acesso facilitado aos artigos científicos publicados no Brasil e no exterior exigem dos profissionais apreciação crítica para identificar se as informações são atuais e de boa qualidade, garantindo assim o uso de pesquisas clinicamente relevantes em ambientes de práticas. Por isso, na busca por integrar as experiências individuais vivenciadas na prática com a melhor evidência clínica disponível a partir de pesquisas sistemáticas, surge a Prática Baseada em Evidência (PBE), movimento científico que tem ganhado força na área da saúde. A PBE é definida como o "processo de sistematicamente descobrir, avaliar e utilizar os resultados da investigação científica contemporânea como base para decisões clínicas" (3). Por isso, a evidência não substitui a habilidade e a experiência individual do profissional, que é quem julga e avalia se a evidência se aplica ao paciente em questão e se pode ser integrada na tomada de decisão clínica (4).

Muitos fisioterapeutas não possuem formação suficiente em metodologia da pesquisa para distinguir 
entre os estudos de alta e baixa qualidade, assim como identificar os pontos relevantes para sua prática clínica na literatura científica. Por isso, em muitos países são oferecidos cursos de formação continuada sobre a implantação da PBE. Além disso, vários cursos de pós-graduação nos Estados Unidos, Austrália, Canadá e Reino Unido oferecem cursos de PBE. No Canadá, por exemplo, todos os programas educacionais têm a PBE como componente essencial dos currículos (5). Contudo, os profissionais ainda enfrentam muitas barreiras limitadoras do uso da literatura científica na prática clínica. Algumas dessas barreiras incluem: a dimensão e a complexidade da literatura científica, o deficiente acesso aos elementos de evidência, organização e educação ineficaz (6).

Até a data da elaboração deste artigo, não havia estudos que descrevessem se os profissionais fisioterapeutas clínicos que não estão ligados à comunidade acadêmica da Grande Florianópolis têm acesso ou utilizam essa produção científica. Por isso, esta pesquisa tem como objetivo averiguar se esses profissionais fisioterapeutas da Grande Florianópolis têm facilidade e habilidade em buscar literatura científica e se eles se baseiam nessa literatura durante sua prática clínica. Além disso, pretende-se identificar se a PBE é considerada uma ferramenta importante na tomada de decisão clínica e quais as principais barreiras para sua implantação no contexto das cidades da Grande Florianópolis. 0 conhecimento gerado com esta pesquisa poderá ajudar as instituições de Ensino Superior, conselhos e profissionais da área acadêmica a adotar estratégias para auxiliar os profissionais de Fisioterapia tanto na procura e quanto na aplicação do conhecimento científico na prática clínica.

\section{Materiais e métodos}

Sujeitos

Participaram do estudo 67 profissionais fisioterapeutas que atuam na região da Grande Florianópolis, nos municípios de São José, Palhoça e Florianópolis. A maioria dos participantes era do sexo feminino (55) e apenas 12 do sexo masculino (Quadro 1).

Os critérios de inclusão no estudo foram: ser fisioterapeuta em atividade no atendimento de pacientes e assistência ao cidadão na área de fisioterapia; exercer essas atividades na região da Grande
Florianópolis nos municípios de São José, Palhoça ou Florianópolis; aceitar participar do estudo respondendo a um questionário. 0 recrutamento dos participantes foi realizado contatando os fisioterapeutas em seus locais de trabalho (hospitais, clínicas, consultórios, ambulatórios, academias de musculação, estúdio de pilates) por meio de visitas ou por telefone, a fim de adquirir seus endereços eletrônicos para envio do questionário. Além disso, a pesquisa foi divulgada nos meios eletrônicos, em grupos sociais formados por fisioterapeutas de Santa Catarina. A participação no estudo foi totalmente voluntária e anônima. Todos os indivíduos foram esclarecidos sobre os objetivos e procedimentos da pesquisa e assinaram o Termo de Consentimento Livre e Esclarecido aprovado pelo Comitê de Ética da Universidade Estadual de Santa Catarina (protocolo n. 158/2011).

Instrumentos de coleta de dados

Foi utilizado um questionário traduzido e adaptado a partir do Evidence-Based Practice Questionnaire (7). Este foi composto por 47 perguntas, fechadas e abertas, compondo três blocos de variáveis: 1) dados sociodemográficos: sexo, idade, tipo de instituição em que se graduou, tempo de graduado, tipo de pós-graduação; 2) campo de trabalho: área de atuação, jornada de trabalho, renda, característica da prática clínica, satisfação; 3) campo do conhecimento: facilidade/habilidade de pesquisar literatura e associá-la com a prática clínica; facilidade/habilidade de acesso a bancos de dados na internet, participação em formação continuada e congressos científicos, conhecimento sobre a PBE. O questionário foi utilizado na forma virtual usando a ferramenta JotForm, que funciona no navegador Web. Trata-se de uma ferramenta de serviço gratuito para a construção de formulários online. Para divulgação, esclarecimento da pesquisa e acesso ao questionário, foi utilizado o aplicativo Google Sites, ferramenta da Web 2.0, que permite a criação de páginas eletrônicas na $W e b$.

\section{Procedimentos}

Os indivíduos foram orientados, por meio de correio eletrônico, a acessar a página eletrônica em que a pesquisa foi apresentada e, somente após assinar o Termo de Consentimento Livre e Esclarecido, eles 
puderam responder ao questionário. Este foi dividido em quatro páginas eletrônicas, a fim de facilitar a leitura e o entendimento. Só era possível finalizar o questionário se todas as questões fossem respondidas.

\section{Análise dos dados}

Trata-se de um estudo descritivo, cujos dados foram codificados e transcritos utilizando-se o software Microsoft Office Excel, versão 2007, e processados no programa estatístico SPSS (Statistical Package for the Social Sciences, versão 17.0). Os dados foram analisados por cálculo de frequência absoluta e relativa, apresentados em gráficos e tabelas para melhor descrição dos resultados.

\section{Resultados}

\section{Dados sociodemográficos}

Os dados sociodemográficos estabelecidos por este estudo encontram-se no Quadro 1. Na relação de profissionais por gênero, houve forte predominância do sexo feminino, com $82,1 \%$, contra $17,9 \%$ do sexo masculino. Além disso, a população de fisioterapeutas entrevistados foi jovem, mantendo-se numa faixa de idade predominantemente entre 20 e 29 anos, representando um total de $73,1 \%$ dos entrevistados. Possivelmente, em consequência da faixa etária, $73,1 \%$ dos entrevistados concluíram a graduação há menos de cinco anos. Grande parte dos participantes $(71,6 \%)$ não possuía título de especialista reconhecido pelo Crefito. Dos pou$\cos (28,4 \%)$ que apresentaram alguma especialização reconhecida, estas foram: Acupuntura, Fisioterapia Dermatofuncional, Ergonomia, Fisioterapia Desportiva e Fisioterapia Traumato-Ortopédica.

A maioria dos fisioterapeutas entrevistados $(53,7 \%)$ graduou-se em instituição privada e apenas 7,5\% tinha o nível de mestrado; nenhum possuía doutorado. Entretanto, 80,6\% dos que não apresentavam esse nível de pós-graduação mostraram-se interessados em adquiri-lo no futuro.

\section{Campo de trabalho}

Aproximadamente $68,7 \%$ dos profissionais entrevistados apresentaram renda mensal entre
$\mathrm{R} \$ 1.000,00$ e $\mathrm{R} \$ 5.000,00,22,4 \%$ afirmaram receber entre $R \$ 500,00$ e $R \$ 1.000,00$, e somente $7,5 \%$ recebem acima de $\mathrm{R} \$ 5.000,00$. Em relação ao grau de satisfação dos fisioterapeutas com a profissão, 40,3\% afirmaram estar satisfeitos, $31,3 \%$ mostraram-se neutros sobre o assunto, $16,4 \%$ mostraram-se poucos satisfeitos e apenas 3\% apresentaram insatisfação.

A maioria dos entrevistados $(85,1 \%)$ não apresentava outra fonte de renda. Dentre os que relataram outra fonte de renda que não a fisioterapia $(14,9 \%)$, as seguintes ocupações foram citadas: confecção de artesanato, venda de cosmético, técnico em informática, consultoria Google, cargo administrativo, comércio e ensino de inglês.

Quadro 1- Dados sociodemográficos

\begin{tabular}{|c|c|c|}
\hline Característica & $\begin{array}{l}\text { Frequência } \\
\text { absoluta (n) }\end{array}$ & $\begin{array}{l}\text { Frequência } \\
\text { relativa (\%) }\end{array}$ \\
\hline \multicolumn{3}{|l|}{ Sexo } \\
\hline Feminino & 55 & 82,1 \\
\hline Masculino & 12 & 17,9 \\
\hline \multicolumn{3}{|l|}{ Idade } \\
\hline $20-29$ anos & 49 & 73,1 \\
\hline 30-39 anos & 17 & 25,4 \\
\hline 40-49 anos & 1 & 1,5 \\
\hline \multicolumn{3}{|c|}{ Há quantos anos concluiu a graduação } \\
\hline Menos de 5 & 49 & 73,1 \\
\hline $5-10$ & 6 & 9,0 \\
\hline $11-15$ & 12 & 17,9 \\
\hline \multicolumn{3}{|c|}{ Tipo de instituição em que se graduou } \\
\hline Pública & 31 & 46,3 \\
\hline Privada & 36 & 53,7 \\
\hline \multicolumn{3}{|c|}{ Maior grau alcançado } \\
\hline Graduação & 37 & 55,2 \\
\hline $\begin{array}{l}\text { Especialização lato } \\
\text { sensu }\end{array}$ & 25 & 37,3 \\
\hline Mestrado & 5 & 7,5 \\
\hline Doutorado & 0 & 0 \\
\hline \multicolumn{3}{|c|}{$\begin{array}{l}\text { Especialidade profissional ou acadêmica reconhecida } \\
\text { pelo Coffito }\end{array}$} \\
\hline Não & 48 & 71,6 \\
\hline Sim & 19 & 28,4 \\
\hline
\end{tabular}

Fonte: Dados da pesquisa. 
As características da prática profissional dos fisioterapeutas entrevistados encontram-se no Quadro 2. A quantidade de pacientes atendidos por dia variou entre os fisioterapeutas entrevistados: $13,4 \%$ dos entrevistados atendem menos de 5 pacientes, $43,3 \%$ atendem entre cinco e dez pacientes, $34,3 \%$ atendem entre 11 e 15 pacientes e $9 \%$ atendem mais de 15 pacientes por dia. Além disso, 47,8\% desses profissionais atendem apenas um paciente por vez e $68,7 \%$ demoram em média 60 minutos por atendimento.

No que se refere à jornada de trabalho semanal, esta variou: menos de 20 horas $(10,4 \%)$, entre 20 e 30 horas (32,8\%), entre 31 a 40 horas $(34,3 \%)$ e acima de 40 horas (22,4\%).

Muitos prestaram serviço de fisioterapia em mais de um ambiente de trabalho; $61,2 \%$ atuaram em apenas um local, 34,3\% atuaram em dois locais e $4,5 \%$ atuaram em três locais distintos. Os ambientes

Quadro 2 - Características do campo de trabalho

\begin{tabular}{lcc}
\hline Característica & $\begin{array}{c}\text { Frequência } \\
\text { absoluta (n) }\end{array}$ & $\begin{array}{c}\text { Frequência } \\
\text { relativa (\%) }\end{array}$ \\
\hline Horas trabalhadas por semana & \\
\hline Menos de 20h & 7 & 10,4 \\
20-30h & 22 & 32,8 \\
31-40h & 23 & 34,3 \\
Acima de 40h & 15 & 22,4 \\
\hline Pacientes atendidos por dia & \\
\hline Menos de 5 & 9 & 13,4 \\
5-10 & 29 & 43,3 \\
11-15 & 23 & 34,3 \\
Acima de 15 & 6 & 9,0 \\
\hline Pacientes atendidos ao mesmo tempo & \\
\hline 1 & 32 & 47,8 \\
2 & 19 & 28,4 \\
3 & 12 & 17,9 \\
Acima de 4 & 4 & 6,0 \\
\hline Duração média do atendimento & \\
\hline Menos de 30 min & 3 & 4,5 \\
$30-40$ min & 15 & 22,4 \\
$40-60$ min & 46 & 4,5 \\
\hline Acima de 60 min & 3 & \\
\hline
\end{tabular}

Fonte: Dados da pesquisa. que concentraram o maior número de atuações foram: contratado de clínica privada $(34,4 \%)$, cuidados domiciliares $(18,8 \%)$ e proprietário de clínica privada $(16,7 \%)$.

Com relação às áreas de atuação, a maioria dos fisioterapeutas entrevistados atua em duas a quatro áreas distintas, sendo que 20,9\% atendem pacientes com problemas ortopédicos, $11,8 \%$ atendem pacientes geriátricos, $10,8 \%$ atendem pacientes com acometimento neurológico, e 9,8\%, acometimentos reumatológicos.

\section{Campo de conhecimento}

As pesquisas atuais em revistas científicas na sua forma impressa não foram significativamente utilizadas pela maioria dos profissionais inquiridos, sendo que 77,6\% deles não têm acesso a esse tipo de informação. Já no que se refere ao acesso à base de dados na internet, a maioria dos profissionais entrevistados relatou acessar esse tipo de literatura tanto em casa como no local de trabalho, representando um total de $49,3 \%$ dos entrevistados. Já 41,8\% informaram que acessam essas informações apenas em casa.

Aproximadamente 40,3\% dos entrevistados afirmaram que quase sempre utilizaram base de dados como LILACS, SciELO, PEDro para pesquisa da literatura científica relevante, utilizando os resultados dessas pesquisas quase sempre em sua prática clínica. Além disso, a maioria $(65,7 \%)$ afirmou ler por mês, em média, de dois a cinco artigos científicos relacionados com a prática clínica.

Quanto ao incentivo do local de trabalho ao uso dos resultados de investigações atuais na prática clínica, 47,8\% dos inquiridos mostraram-se neutros no assunto, $23,9 \%$ concordaram e $16,4 \%$ concordaram totalmente. Cerca de $35,8 \%$ dos entrevistados mostraram-se neutros, 20,9\% discordaram e 25,4\% discordaram totalmente do fato de que eles têm recebido treinamento formal em estratégias de busca para encontrar literatura online relevante para a prática clínica. Já no que concerne ao recebimento de treinamento formal em avaliação crítica de artigos científicos durante a formação acadêmica, 43,3\% dos entrevistados concordam, 19,4\% concordam totalmente, enquanto que $14,9 \%$ discordaram ou mostraram-se neutros. A maioria dos entrevistados $(59,7 \%)$ mostrou-se confiante em sua capacidade de análise crítica de artigos científicos, assim como 
em sua capacidade de encontrar artigos científicos relevantes para responder às suas questões clínicas (Gráfico 1).

A maioria dos profissionais fisioterapeutas entrevistados (83,6\%) afirmou participar de cursos de educação continuada ao menos uma vez no ano. Além disso, mais da metade dos entrevistados $(59,7 \%)$ afirmou participar de congressos científicos.

Aproximadamente $75 \%$ dos entrevistados confirmaram o conhecimento sobre a Prática Baseada na Evidência. A maioria deles (50\%) concordou ter aprendido as bases para a PBE como parte de sua preparação acadêmica, além de concordarem (48\%) e concordarem totalmente (40\%) com o fato de que a aplicação da PBE é necessária para a prática da fisioterapia. Por isso, 68\% dos inquiridos afirmaram usar a PBE em sua prática clínica diária (Gráfico 2).

Por volta de $56 \%$ dos entrevistados mostraram-se neutros no que se refere à afirmação de que a PBE não leva em conta as limitações da prática clínica de cada profissional, enquanto $22 \%$ concordaram com essa afirmação. De forma semelhante, $76 \%$ dos entrevistados mostraram-se neutros na afirmação de que a PBE não leva em conta as preferências do paciente. Em contrapartida, $66 \%$ dos entrevistados concordaram que a PBE ajuda a tomar decisões sobre o tratamento dos pacientes.
Os entrevistados mostraram-se neutros (44\%) em relação à afirmação de que a incorporação da PBE coloca uma responsabilidade demasiada sobre o fisioterapeuta e 34\% discordaram dessa afirmação. Além disso, $44 \%$ mostraram-se neutros e $40 \%$ discordaram que seu ganho financeiro aumentará ao incorporar a PBE em sua prática clínica (Gráfico 2).

A maioria dos entrevistados (46\%) reiterou ser necessário aumentar o uso de evidências científicas em sua prática clínica diária. Além disso, $60 \%$ concordou estar interessado em aprender ou aprimorar as habilidades necessárias para incorporar PBE em sua prática. Também, $40 \%$ concordou ou $34 \%$ mostraram-se neutros com relação ao fato de estarem faltando fortes evidências científicas para a maioria das intervenções utilizadas por eles em seus pacientes (Gráfico 2).

\section{Barreiras}

O tempo insuficiente foi considerado a barreira mais importante para o uso de evidências na prática clínica, sendo indicada por $48,8 \%$ dos inquiridos, além de ter sido considerada por $11,6 \%$ dos entrevistados como segunda e terceira barreiras. A falta de generalização dos achados da literatura científica para a população de pacientes específica de cada fisioterapeuta

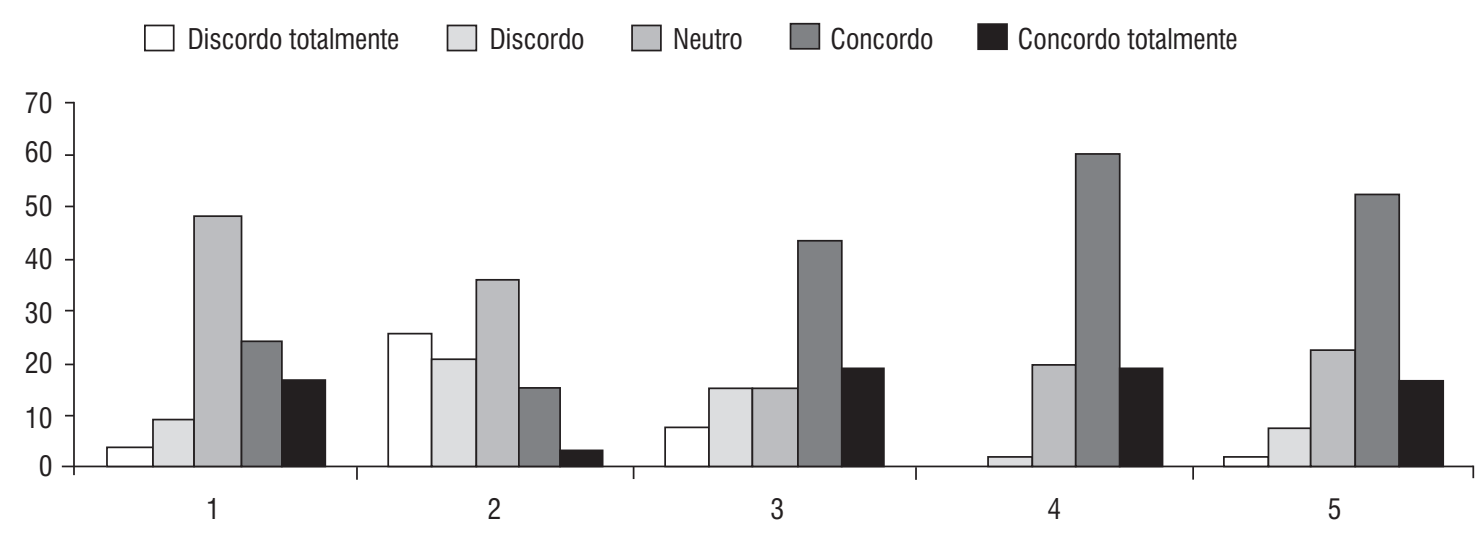

Gráfico 1 - Facilidades e habilidades relacionadas à obtenção e à avaliação da literatura científica pelos profissionais fisioterapeutas entrevistados

Legenda: 1 = Local de trabalho incentiva 0 uso dos resultados de investigações atuais na prática clínica; 2 = Treinamento em estratégias de busca para encontrar literatura online relevante para a minha prática; 3 = Treinamento formal em avaliação crítica de artigos científicos como parte de minha preparação acadêmica; 4 = Confiança na capacidade de análise crítica de artigos científicos; 5 = Confiança na capacidade de encontrar artigos científicos relevantes para responder às minhas questões clínicas.

Fonte: Dados da pesquisa. 
Facilidades e habilidades do fisioterapeuta na procura, interpretação e aplicação do conhecimento científico na prática clínica

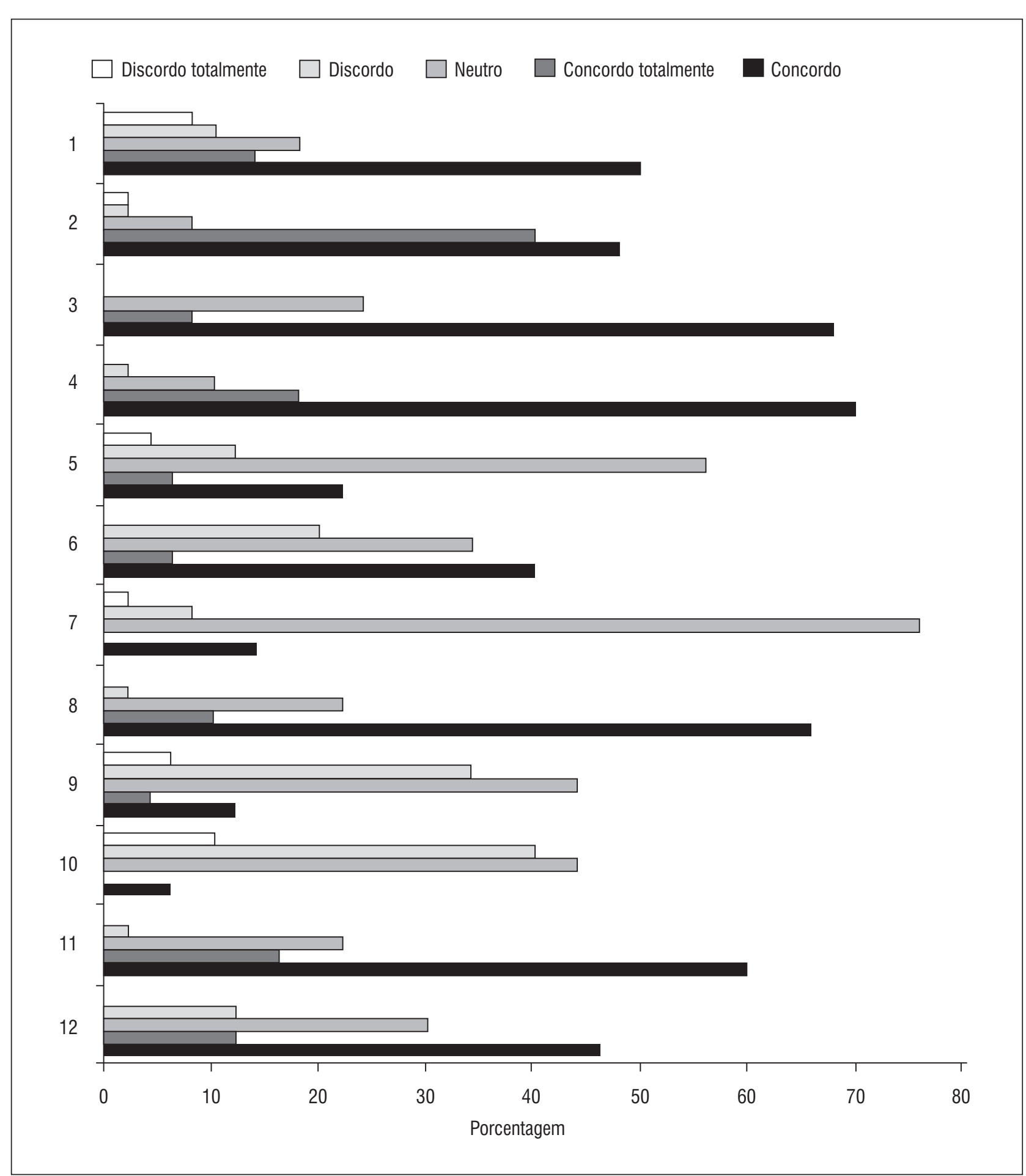

Gráfico 2 - Distribuição das respostas relativas à educação, conhecimento e habilidades associadas com PBE

Legenda: 1 = Aprendi as bases para a PBE, como parte de minha preparação acadêmica; 2 = Aplicação da PBE é necessária para a prática da fisioterapia; 3 = Uso PBE na prática diária; 4 = PBE melhora a qualidade do atendimento ao paciente; $5=$ PBE não leva em conta as limitações da minha prática clínica; 6 = Estão faltando fortes evidências científicas para a maioria das intervenções que eu uso nos paciente; 7 = PBE não leva em conta as preferências do paciente; 8 = PBE ajuda a tomar decisões sobre 0 tratamento dos pacientes; 9 = A incorporação da PBE coloca uma responsabilidade demasiada sobre o fisioterapeuta; $10=$ Meu ganho financeiro irá aumentar se eu incorporar a PBE na minha prática; 11 = Interesse em aprender ou aprimorar as habilidades necessárias para incorporar PBE em minha prática; 12 = Precisa aumentar o uso de evidências científicas em minha prática diária. Fonte: Dados da pesquisa. 
foi considerada como a segunda e a terceira barreiras mais importante por $32,6 \%$ e por $23,3 \%$ dos inquiridos, respectivamente. Além disso, a falta de fontes de informação foi classificada como segunda barreira por 25,6\% dos fisioterapeutas e a incapacidade de aplicar os resultados aos pacientes individuais como características únicas foi escolhida por $16,3 \%$ dos inquiridos como terceira barreiras (Gráfico 3).

\section{Discussão}

A amostra foi formada por profissionais jovens, com forte predomínio do sexo feminino, tendência já reportada em estudos anteriores com fisioterapeutas $(7,8,9)$. A maioria dos entrevistados tem uma renda mensal entre $\mathrm{R} \$ 1.000,00$ e $\mathrm{R} \$ 5.000,00$; poucos possuem outra fonte de renda além da alcançada por meio da fisioterapia, e grande parte dos entrevistados mostrou-se satisfeita com a profissão. Apesar de não existir lei que fixe o piso salarial dos fisioterapeutas, em 2009 foi lançado um Projeto de Lei que busca fixar o piso salarial em $\mathrm{R} \$ 4.650,00$ para uma jornada de trabalho de 30 horas semanais, o qual está tramitando e aguarda aprovação (10). Enquanto não existe normativa estipulando o piso da categoria, a Federação Nacional de Fisioterapia e Terapia Ocupacional (Fenafito) adota como referência o piso de $\mathrm{R} \$ 1.870,00$, homologado com a Delegacia Regional do Trabalho pelo Sindicato dos Fisioterapeutas e Terapeutas Ocupacionais no Estado e São Paulo (Sinfito/SP) (11). Portanto, os fisioterapeutas entrevistados da Grande Florianópolis estão em uma faixa salarial compatível com o esperado, porém, com uma carga horária elevada, já que muitos trabalham acima de 30 horas semanais. Sendo assim, cabe às associações, autoridades da profissão e autoridades governamentais intervirem a fim de oferecer salário e carga horária justos.

A ortopedia foi a área de maior atuação dos profissionais fisioterapeutas entrevistados, coincidindo com a área predominante na Revista Brasileira de Fisioterapia e na revista Fisioterapia em Movimento, representando $36,2 \%$ das publicações, seguida por pneumologia $(11,4 \%)$ e neurologia $(9,7 \%)(12)$. Além disso, o autor observou aumento do número de publicações entre 2005 e 2009 nessas revistas com predominância de estudos vinculados à área de ortopedia. A neurologia foi considerada pelos fisioterapeutas entrevistados como a terceira maior área de atuação, também sendo considerada por Virtuoso et al. (12) como a terceira de maior publicação.

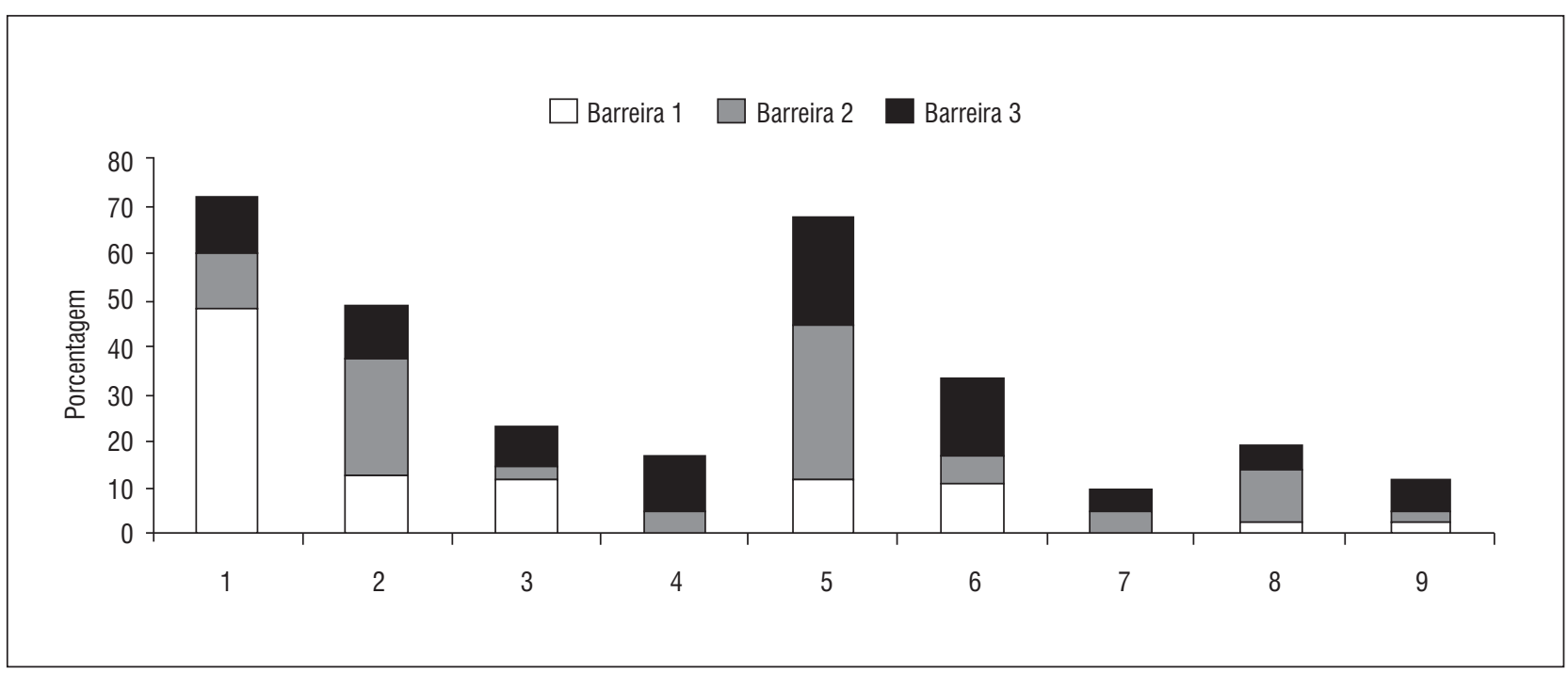

Gráfico 3 - Barreiras 1, 2 e 3 mostradas em ordem de importância (primária, secundária e terciara) para 0 uso da literatura científica na prática clínica dos profissionais fisioterapeutas entrevistados

Legenda: 1 = Tempo insuficiente; 2 = Falta de fontes de informação; 3 = Falta de habilidades em pesquisar; 4 = Fraca capacidade de apreciar criticamente a literatura científica; 5 = Falta de generalização dos achados da literatura científica para a minha população de pacientes; 6 = Incapacidade para aplicar os resultados da investigação científica para pacientes com características únicas; 7 = Falta de compreensão da análise estatística; 8 = Falta de apoio coletivo entre meus colegas de trabalho; 9 = Falta de interesse.

Fonte: Dados da pesquisa. 
Os resultados demonstraram que os fisioterapeutas da Grande Florianópolis têm uma relação positiva com PBE, uma vez que consideraram que o uso da literatura científica auxilia na tomada de decisão, que a qualidade do atendimento ao paciente melhora quando a evidência é utilizada e que ela é uma ferramenta importante para a Fisioterapia. Em uma média mensal, a grande maioria lê de dois a cinco artigos relacionados à prática clínica. A taxa de leitura, somente razoável, pode estar associada ao fato de que a maioria considerou que faltam evidências científicas para boa parte das intervenções realizadas em seus pacientes. Isso porque, como observamos, $43,3 \%$ e $34,3 \%$ dos fisioterapeutas entrevistados atendem, respectivamente, de 5-10 a 11-15 pacientes por dia (Quadro 2), o que implicaria um grande número de casos por mês e, em consequência, maior busca pela literatura científica. Outra razão para essa taxa de leitura é que, atualmente, existem cerca de 12 revistas brasileiras de fisioterapia, e essa quantidade pode não ser suficiente para atender a demanda de publicação dos conhecimentos científicos gerados e formar um banco de dados que permita o ensino da fisioterapia baseada em evidências (13). Além disso, grande parte dos artigos publicados na área de fisioterapia é internacional, o que exige conhecimento de outro idioma, principalmente o inglês, predominante nas publicações de melhor qualidade.

As análises de literatura apontam baixa qualidade das pesquisas publicadas na área de fisioterapia (14). Por exemplo, uma pesquisa por meio da escala de qualidade PEDro, na qual foram analisados 50 estudos controlados randomizados de fisioterapia publicados em português, constatou que a pontuação média dos estudos foi de 3,4 pontos de um possível total de 10 pontos. Além disso, 84\% desses estudos apresentaram nota 4 ou inferior (15). Portanto, esses estudos de baixa qualidade metodológica podem proporcionar informações com pouca validade científica, o que pode influenciar equivocadamente a prática clínica da fisioterapia.

Todavia, a mera publicação de pesquisa de alta qualidade não resultará no conhecimento para traduzi-la facilmente na prática clínica. Talvez por isso, muitos fisioterapeutas tenham mostrado interesse em aprimorar as habilidades para incorporar a PBE na sua prática diária. Além disso, a maioria participou de formação continuada e de congressos científicos. A participação nesses eventos deve ser considerada pelo fisioterapeuta como parte importante de seu preparo para implementar a PBE em sua vida profissional (16).

Os resultados mostraram que a maioria dos fisioterapeutas utilizou a internet como principal ferramenta na busca de literatura científica; as bases de dados mais citadas foram LILACS, SciELO e PEDro. 0 acesso à internet favorece a utilização de bases de dados digitais e recursos de informação, sendo uma alternativa viável para apoiar a prática baseada na evidência (17). Além disso, o uso de filtros metodológicos é uma estratégia simples que pode melhorar habilidades de fisioterapeutas para identificar ensaios de alta qualidade $(4,18)$.

Embora a maioria dos entrevistados tenha admitido acessar internet e base de dados online tanto em casa quanto no local de trabalho, muitos se mostraram neutros quanto ao incentivo do seu local de trabalho ao uso do resultado da investigação na prática clínica. 0 uso eficiente do tempo é uma necessidade nos locais de trabalho, sendo importante que os profissionais tenham os recursos necessários para acessar as evidências. Vale ressaltar que fontes de informações acerca da prática clínica estão cada vez mais disponíveis (19). Por exemplo, a maioria dos médicos da rede pública de saúde nos grandes hospitais da Grande Florianópolis (Hospital Celso Ramos e Regional de São José) conta com biblioteca e acesso a computadores e à internet. Portanto, bibliotecas virtuais, bases eletrônicas de dados e periódicos eletrônicos de boa qualidade precisam ser disponibilizados aos fisioterapeutas em seus locais de trabalho, de forma a poder garantir a informação privilegiada. No caso de clínicas e hospitais particulares, essas informações, cujo custo é considerável, poderiam ser negociadas com o proprietário, com os convênios de saúde, ou com os dois.

Similarmente ao estudo de Jette et al. (7), os entrevistados em nosso estudo não tinham certeza (neutros) se a PBE levaria em conta as limitações em empregar a evidência na sua prática ou se ela atenderia às preferências de seus pacientes. Dessa forma, os fisioterapeutas inquiridos neste estudo podem tomar decisões dicotomizadas em relação ao PBE, ora atendendo ao que é indicado pela literatura científica, ora atendendo às condições da prática. Ensaios clínicos e revisões sistemáticas podem fornecer informações relativamente imparciais sobre os efeitos do tratamento sobre o paciente, contudo, a experiência clínica e a intuição do profissional podem ser capazes de discriminar entre os pacientes que 
responderão ou não a um determinado tratamento (20). Além disso, a PBE requer o uso consciente das melhores evidências de estudos clínicos para orientar a escolha do tratamento aliado à experiência prática e à escolha da paciente (21). Assim, mesmo diante do melhor nível de evidência disponível, não se pode excluir o uso da prudência, experiência profissional e a necessidade do paciente na escolha de um tratamento adequado $(21,22)$. Por isso, devem ser integradas as decisões clínicas aos valores do paciente, isto é, as preferências originais, preocupações e expectativas que cada paciente traz para um encontro clínico (21).

O tempo insuficiente foi a principal barreira para o uso de literatura científica na prática clínica. Isso porque, mesmo com a prática e os recursos ideais, o processo de encontrar e apreciar criticamente a melhor evidência que responda a uma pergunta clínica geralmente leva um tempo considerável (20). Além disso, o tempo para leitura é fundamental para a avaliação e a adequada aplicação dos resultados da investigação para a prática clínica (23). Portanto, é imprescindível que os fisioterapeutas da Grande Florianópolis diminuam sua jornada de trabalho e o número de pacientes atendidos por dia, para que, dessa forma, tenham mais tempo para o estudo de seus pacientes e da literatura científica. É compreensível que a excessiva jornada de trabalho e o grande número de pacientes atendidos por dia estejam relacionados aos ganhos financeiros; entretanto, as associações, os conselhos e as autarquias de classe têm que continuar na luta por um salário ou honorários por atendimento (principalmente aqueles recebidos por convênio) dignos da responsabilidade do fisioterapeuta, atrelados a uma jornada de trabalho respeitável.

Sabe-se que este estudo tem limitações: a primeira é o número limitado de sujeitos na amostra. Nesse sentido, sugere-se a realização de novas investigações sobre a relação dos profissionais fisioterapeutas com a PBE com uma amostragem maior e representativa da população brasileira. Para tanto, é imprescindível a mobilização de vários profissionais de diferentes localidades e grande apoio das instituições de classe (conselhos e associações). Em segundo lugar, duas questões no questionário podem ser readaptadas para refletir melhor algumas características do fisioterapeuta brasileiro: 1) inclusão no questionário da opção de resposta "não atendo por convênio", questão 17; e 2) diminuição da distancia entre as faixas salariais, questão 18.

\section{Conclusão}

A maioria dos fisioterapeutas da Grande Florianópolis são jovens, recém-graduados e tendem a expressar atitudes positivas quanto ao uso da PBE. Além disso, confiam em suas habilidades relacionadas ao acesso e à apreciação crítica da informação. Eles se declararam interessados em aumentar suas competências e a quantidade de elementos/informações utilizados em suas práticas clínicas. A falta de tempo foi a barreira mais importante para o uso da PBE. Dessa maneira, medidas no sentido de propiciar facilidades e habilidades no desenvolvimento do conhecimento científico para os fisioterapeutas devem ser implementadas, levando-se em consideração o fator tempo.

\section{Referências}

1. Conselho Regional de Fisioterapia e Terapia Ocupacional - Crefito. Leis e atos normativos das profissões de fisioterapeuta e do terapeuta ocupacional. Porto Alegre: CREFITO; 2001.

2. Coury H, Vilella I. Profile of the brazilian physical therapy researcher. Rev Bras Fisioter. 2009;13(4):356-63.

3. Guyatt G. Evidence-based medicine - a new approach to teaching the practice of medicine. Jama-J Am Med Assoc. 1992;268(17):2420-5.

4. Sackett DL. Evidence-based medicine: how to practice and teach EBM. Edinburgh; New York: Churchill Livingstone; 2000.

5. Bennett S, Townsend L, Marcini M, Taylor C. Evidence-based practice in occupational therapy: international initiatives. World Federation of Occupational Therapists; 2006 [cited 2006 Jan. 21]. Available at: http:// espace.library.uq.edu.au/view/UQ:79210.

6. Haynes B, Haines A. Getting research findings into practice: barriers and bridges to evidence based clinical practice. Br Med J. 1998;317(7153):273-6.

7. Jette DU, Bacon K, Batty C, Carlson M, Ferland A, Hemingway RD, et al. Evidence-based practice: beliefs, attitudes, knowledge, and behaviors of physical therapists. Phys Ther. 2003;83(9):786-805. 
8. Badaró AFV, Guilhem D. Perfil sociodemográfico e profissional de fisioterapeutas e origem das suas concepções sobre ética. Fisioter Mov. 2011;24(3):445-54.

9. Tombini FF. Fisioterapia: considerações sobre uma ciência em construção. Ijuí: Ed. Unijuí; 2002.

10. Conselho Federal de Fisioterapia - Coffito. Principais Projetos de Leis. [acesso 5 maio 2012]. Disponível em: http://www.coffito.org.br/PROJETOS\%20DE\%20 LEI/Projetos\%20Site\%2016\%2004\%202012.pdf.

11. Federação Nacional de Fisioterapia e Terapia Ocupacional - Fenafito. Referência salarial. Brasília [acesso 5 maio 2012]. Disponível em: http://www.fenafito. com.br/main.asp?link=noticia\&id=28.

12. Virtuoso JF, Haupenthal A, Pereira ND, Martins CP, Knabben RJ, Andrade A. A produção de conhecimento em fisioterapia: análise deperiódicos nacionais (1996 a 2009). Fisioter Mov. 2011;24(1):173-80.

13. Calvalcante CCL, Rodrigues ARS, Dadalto TV, Silva EB. Evolução científica da fisioterapia em 40 anos de profissão. Fisioter Mov. 2011;24(3):513-22.

14. Green S, Buchbinder R, Hetrick S. Acupuncture for shoulder pain. Cochrane Database Syst Rev. 2005;2.

15. Shiwa SR, Costa LOP, Moser ADL, Aguiar IC, Oliveira LVF. PEDro: a base de dados de evidências em Fisioterapia. Fisioter Mov. 2011;24(3):523-33.

16. Bury TJ, Mead JM. Evidence based healthcare: a practical guide for therapists. Oxford: Butterworth-Heinemann; 2000.

17. Brown SR, Roush JR, Lamkin AR, Perrakis R, Kronenfeld MR. Evaluating the professional libraries of practicing physical therapists. J Med Libr Assoc. 2007;95(1): 64-9.
18. Guyatt GH, Sackett DL, Cook DJ. User's guides to the medical literature: II. how to use an article about therapy or prevention a. are the results of the study valid? Jama-J Am Med Assoc. 1993;270(21):2598-601.

19. Morris J. Evidence-based Practice: the way forward. Physiotherapy. 2003;89(6):330-1.

20. Herbert R, Sherrington C, Maher C, Moseley AM. Evidence-based practice - imperfect but necessary. Physiother Theory Pract. 2001;17(3):201-11.

21. Herbert R. Practical evidence-based physiotherapy. Edinburgh; New York: Elsevier Butterworth Heinemann; 2005.

22. Sackett DL, Rosenberg WMC, Gray JAM, Haynes RB, Richardson WS. Evidence based medicine: what it is and what it isn't - It's about integrating individual clinical expertise and the best external evidence. $\mathrm{Br}$ Med J. 1996;312(7023):71-2.

23. Salbach NM, Guilcher SJT, Jaglal SB, Davis DA. Factors influencing information seeking by physical therapists providing stroke management. Phys Ther. 2009;89(10):1039-50.

Recebido: 02/06/2012

Received: 06/02/2012

Aprovado: $10 / 11 / 2012$

Approved: 11/10/2012 